\title{
A NEW ULTRALUMINOUS X-RAY SOURCE IN THE NEARBY EDGE-ON SPIRAL NGC 891
}

\author{
Edmund J. Hodges-Kluck, Joel N. Bregman, Jon M. Miller, and Eric Pellegrini \\ Department of Astronomy, University of Michigan, Ann Arbor, MI 48109, USA; hodgesk1@umich.edu \\ Received 2011 December 15; accepted 2012 February 9; published 2012 February 24
}

\begin{abstract}
We report the discovery of a new candidate ultraluminous X-ray source in the nearby edge-on spiral galaxy NGC 891. The source, which has an absorbed flux of $F_{x} \sim 1 \times 10^{-12} \mathrm{erg} \mathrm{s} \mathrm{cm}^{-2}$ (corresponding to an $L_{x} \gtrsim$ $10^{40} \mathrm{erg} \mathrm{s}^{-1}$ at $9 \mathrm{Mpc}$ ), must have begun its outburst in the past five years as it is not detected in prior X-ray observations between 1986 and 2006. We try empirical fits to the XMM-Newton spectrum, finding that the spectrum is fit very well as emission from a hot disk, a cool irradiated disk, or blurred reflection from the innermost region of the disk. The simplest physically motivated model with an excellent fit is a hot disk around a stellar-mass black hole (a super-Eddington outburst), but equally good fits are found for each model. We suggest several follow-up experiments that could falsify these models.
\end{abstract}

Key words: accretion, accretion disks - galaxies: individual (NGC 891) - X-rays: binaries

Online-only material: color figures

\section{INTRODUCTION}

Ultraluminous X-ray sources (ULXs) are non-nuclear X-ray sources with luminosities $L_{X} \gtrsim 10^{39} \mathrm{erg} \mathrm{s}^{-1}$ (Fabbiano 1989). These sources are interesting because their luminosities exceed the Eddington limit for a $10 M_{\odot}$ black hole, suggesting that they are either "intermediate mass" black holes (IMBHs) of $M_{\mathrm{BH}} \sim 10^{2}-10^{4} M_{\odot}$ (Colbert \& Mushotzky 1999) or stellarmass black holes seen during a special time (super-Eddington accretion; see, e.g., Gladstone et al. 2009, hereafter GRD09) or at a special angle (i.e., non-isotropic $L_{X}$; King et al. 2001). In any case, they are important sources for studying black hole physics. For a recent review, see Feng \& Soria (2011).

In this Letter, we report the appearance of a new ULX candidate in the nearby isolated, edge-on spiral galaxy NGC 891. The galaxy is thought to be a Milky Way analog in luminosity (de Vaucouleurs et al. 1991) and color (van der Kruit \& Searle 1981), and, like the Galaxy, it is a barred spiral (e.g., Garcia-Burillo \& Guelin 1995). In Section 2, we describe the $X M M$-Newton detection and other observations, in Section 3 we attempt empirical fits to the X-ray spectrum, and in Section 4 we discuss our results in context.

Throughout this Letter, we use a Galactic column of $N_{\mathrm{H}}=6.5 \times 10^{20} \mathrm{~cm}^{-2}$ (Kalberla et al. 2005) as well as the Wilkinson Microwave Anisotropy Probe cosmology $\left(H_{0}=\right.$ $71 \mathrm{~km} \mathrm{~s}^{-1} \mathrm{Mpc}^{-1}, \Omega_{\Lambda}=0.73$, and $\Omega_{m}=0.27$; Spergel et al. 2007). NGC 891 is about 9-10 Mpc away (Temple et al. 2005; Tully et al. 2009), and we adopt $d \approx 9 \mathrm{Mpc}$.

\section{A NEW CANDIDATE ULX}

A new bright source was discovered in a 133 ks XMM-Newton exposure of NGC 891 (obsID 0670950101) with an aperture-corrected 0.3-10 keV EPIC-pn flux of $F_{X}=$ $1.6 \times 10^{-12} \mathrm{erg} \mathrm{s}^{-1} \mathrm{~cm}^{-2}$ (Figure 1). An inspection of prior X-ray observations between 1994 and 2006, including high-resolution Chandra and ROSAT observations, demonstrates that this source had not yet been detected at sensitivities down to $F_{X} \lesssim$ $10^{-15} \mathrm{erg} \mathrm{s}^{-1} \mathrm{~cm}^{-2}$ in the $0.3-10 \mathrm{keV}$ band. A 2 ks Chandra observation (obsID 14376) indicates source coordinates (J2000)
$02: 22: 33.45 \pm 0.03+42: 20: 26.8 \pm 0.5$ after processing with the sub-pixel EDSER algorithm (Li et al. 2004).

Flux monitoring with the Swift X-Ray Telescope (XRT, target ID 35869) indicates an overall decay with some hint of variability (Figure 2), but further monitoring is necessary to establish a decay. The Swift values include a minor correction for contamination by a nearby X-ray binary with a separation of about the Swift point-spread function (PSF), but Poisson noise is the dominant source of uncertainty.

Follow-up optical observations, including concurrent $X M M$-Newton optical monitor and Swift/UVOT exposures as well as a snapshot with the Mayall $4 \mathrm{~m}$ telescope at Kitt Peak National Observatory, detect no optical counterpart to a limiting magnitude $V \sim 20$ mag. NGC 891 is regularly monitored for supernova candidates, with no detections in the past decade and regular exposures in the past six months (A. Filippenko 2011, private communication). Likewise, a follow-up $5 \mathrm{GHz}$ observation with the Expanded Very Large Array (EVLA) at a sensitivity of $15 \mu \mathrm{Jy} \mathrm{bm}^{-1}$ (for a beam size $\theta \sim 10 \operatorname{arcsec}$ ) found no counterpart. Diffuse emission from NGC 891 with a flux of $F_{v} \approx 80 \mu \mathrm{Jy} \mathrm{bm}^{-1}$ is detected at this position, meaning any future attempt requires higher resolution.

Spectral fits (Section 3) indicate an absorbing column several times higher than the Galactic value, suggesting an extragalactic origin. The source is coincident with the disk of NGC 891, but it may be a background quasar or blazar. Such a source detected in the X-rays ought to be associated with a bright optical/UV counterpart with a flux density within an order of magnitude of the X-rays (see, e.g., Shang et al. 2011), and we would expect rapid variability in the Swift monitoring for a blazar. The absence of these signatures suggests a physical association with NGC 891, but this is not yet certain.

If the source is in NGC 891, the $0.3-10 \mathrm{keV}$ fluxes indicate (absorbed) luminosities between (5-15) $\times 10^{39} \mathrm{erg} \mathrm{s}^{-1}$, suggesting a ULX or supernova. Since we have seen no radio counterpart, a normal Type Ia or II supernova is disfavored. Although we might also expect a radio counterpart to a ULX, the radio luminosities expected are substantially smaller: using the "fundamental plane" expression for radio luminosity as a function of black hole mass and X-ray luminosity in Merloni et al. (2003), we expect $L_{\text {radio }}<10^{34} \mathrm{erg} \mathrm{s}^{-1}$ for a $10^{4} M_{\odot}$ black 


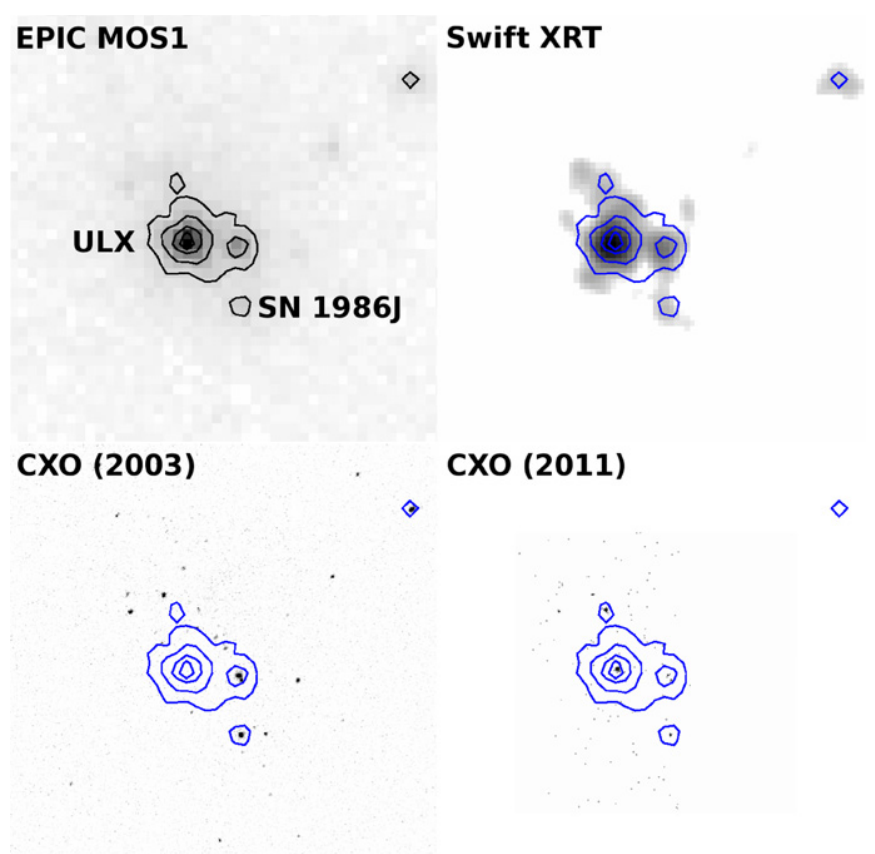

Figure 1. 0.3-10 keV X-ray maps of the field. Top left: EPIC-MOS1 image with bright contours of the same (overlaid on other images). Top right: combined Swift/XRT image from our monitoring campaign ( $21 \mathrm{ks})$, smoothed by 3 pixels. Bottom left: 2003 Chandra image (120 ks). Bottom right: 2011 Chandra snapshot $(2 \mathrm{ks})$. Note the additional new source to the north also seen by $X M M-N e w t o n$ has a $0.3-10 \mathrm{keV} F_{X} \sim 2 \times 10^{-13} \mathrm{erg} \mathrm{s}^{-1} \mathrm{~cm}^{-2}$.

(A color version of this figure is available in the online journal.)

hole. Assuming synchrotron emission near $1 \mathrm{GHz}$ with a spectral index $\alpha=0.7$, the flux density from such a source is at most $\sim 50 \mu \mathrm{Jy}$, already below the diffuse radio halo in the 10 arcsec EVLA beam. The flux would be even less for smaller black holes. The radio non-detection is therefore inconsistent with normal supernovae and consistent with a black hole. Therefore, the source appears to be a new ULX. Hereafter, we refer to it as NGC 891 ULX1. The most recent X-ray observation prior to the XMM-Newton exposure places an upper bound of five years on the outburst.

Archival Hubble Space Telescope (HST) Advanced Camera for Surveys (ACS) HRC and WFC images from 2004 (obsID 9414) reveal a possibly nearby source within an arcsecond of the X-ray position, but not directly associated (Figure 3). Based on centroids of bright sources in the field, the HST astrometry is uncertain to within 0.5 arcsec. The source is faint with an FWHM diameter of $\sim 0.23$ arcsec (the PSF is about 0.1 arcsec at FWHM), and may be a star cluster. In the F555W filter, it has an ST magnitude of $\sim 24.3 \mathrm{mag}\left(M_{\mathrm{F} 555 \mathrm{~W}} \sim-5.7 \mathrm{mag}\right.$, corrected for Galactic $A_{V} \sim 0.22 \mathrm{mag}$ ), and in the F814W filter it is greater than $23.5 \mathrm{mag}\left(M_{\mathrm{F} 814 \mathrm{~W}}>-6.4 \mathrm{mag}\right.$, corrected for Galactic $A_{I} \sim 0.12 \mathrm{mag}$ ). This source is too dim to be seen in the optical data described above. If it is a cluster, the ULX may be physically related (Zezas \& Fabbiano 2002).

\section{SPECTRAL FITS}

We extracted spectra in the $0.3-10 \mathrm{keV}$ band from the EPIC-pn and MOS cameras aboard XMM-Newton using standard Scientific Analysis System recipes. We excluded periods of background flaring during the first $10 \mathrm{ks}$ and last $30 \mathrm{ks}$, leaving $92 \mathrm{ks}$ of good time intervals. Because the field is crowded, we extracted spectra only from within a aperture with
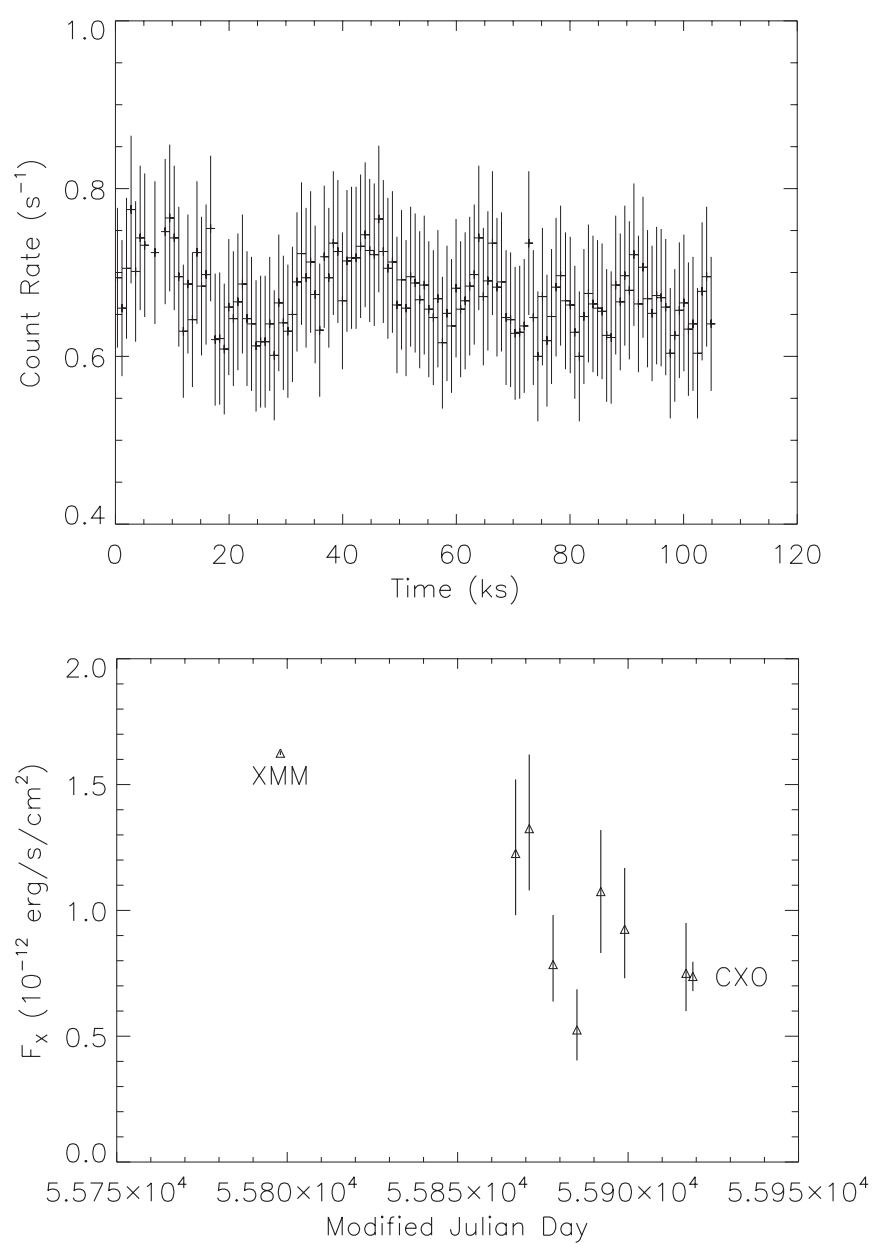

Figure 2. Top: combined synchronized EPIC light curve binned to $800 \mathrm{~s}$ in GTIs. Bottom: XMM-Newton, Swift, and Chandra fluxes measured since 2011 August with $90 \%$ error bars.

$r=25$ arcsec (corresponding to about $80 \%$ encircled energy at $1.5 \mathrm{keV}$ ), but reported fluxes are aperture corrected. The pn count rate in this aperture is $\sim 0.45$ counts $s^{-1}$, and the task epatplot shows a negligible pile-up fraction. The MOS spectra are likewise not piled up.

A two-sided Kolmogorov-Smirnov test finds that the light curve (Figure 2) is incompatible with a constant flux $(P<$ $10^{-6}$ ), but variability is weak, with a fractional rms in $100 \mathrm{~s}$ bins of only $13 \%$ and no evidence for quasi-periodic oscillation in the combined, synchronized light curve.

The spectrum is a featureless continuum aside from the effects of the absorbing column. In this section, we describe empirical model fits using XSPEC version 12.7.0 (Arnaud 1996). Each spectrum is binned by 20 counts and we use the $\chi^{2}$ goodnessof-fit statistic. An acceptable model is one that is not rejected at $95 \%$ probability by this metric. In comparing models that fail the regularity conditions for the $F$-test (Protassov et al. 2002), we calibrate the $F$-test in a method similar to the "parametric bootstrapping" described in Protassov et al. (2002) by using the best-fit model (the null model) to generate a large number (100-1000) of fake spectra with the XSPEC "fakeit" tool for the same exposure time. We fit each simulated spectrum with the null model and the test model to obtain a distribution of $\delta \chi^{2}$, which we compare to the $\delta \chi^{2}$ value for the data. All of our spectral fits incorporate a photoelectric absorption component with $N_{\mathrm{H}}$ frozen to the Galactic value, which is not listed with our 


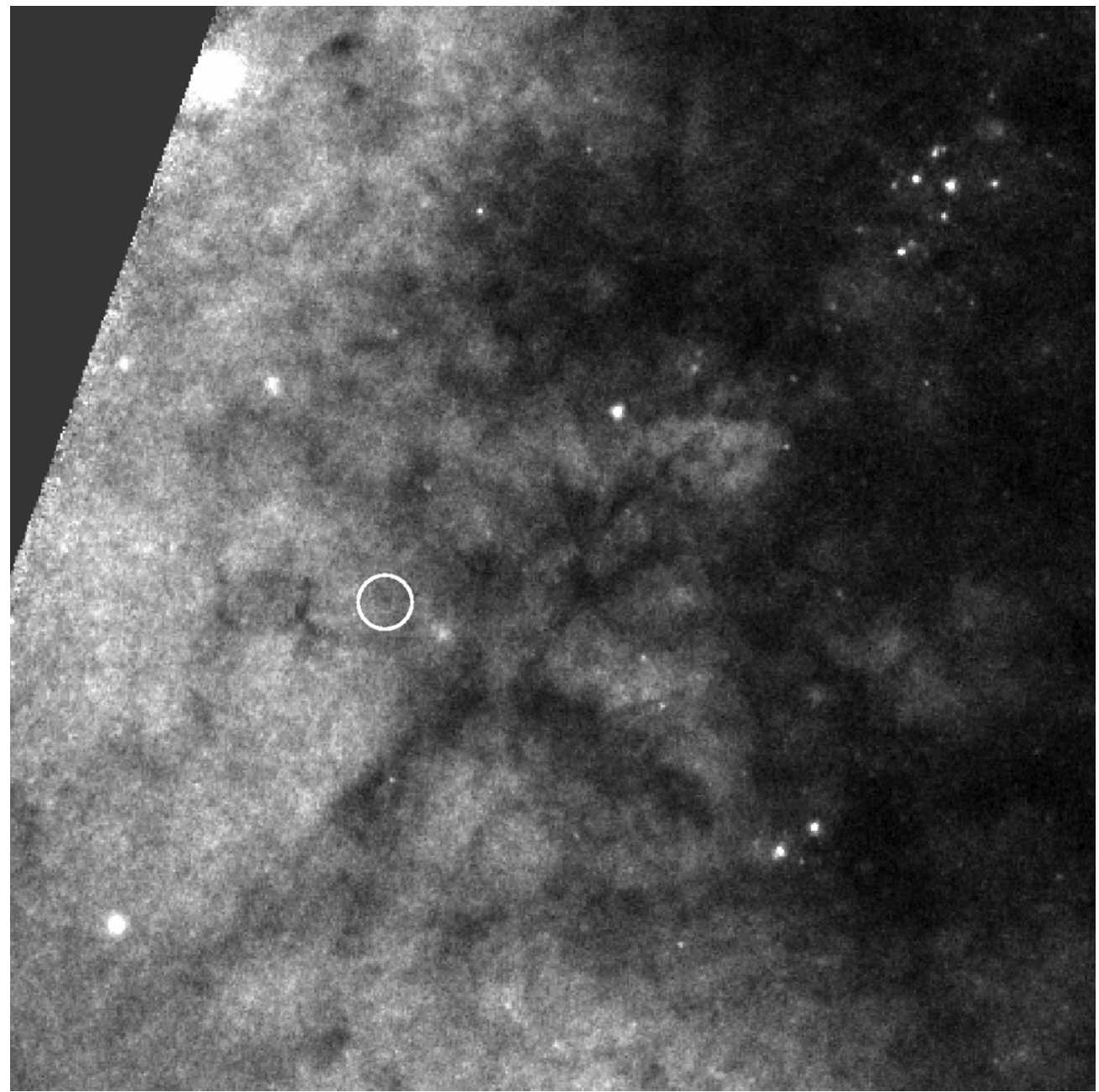

Figure 3. 2004 January 17 HST ACS HRC image of NGC 891 in the F555W filter ( $\sim 5400$ Å). Lighter color is positive emission. The 0 '. 5 ULX position error circle is overplotted, corresponding to $25 \mathrm{pc}$ across.

model parameters (Table 1), as well as an absorption component that is free to vary. We use TBABS with the Wilms et al. (2000) abundances.

The simplest models that have been applied to ULX spectra are absorbed power laws and thermal bremsstrahlung. Although formally a poor fit $\left(\chi^{2} /\right.$ dof $\left.=1860.2 / 1171\right)$, the single power-law model (TBABS*POWERLAW) with $\Gamma=2.24_{-0.02}^{+0.01}$ is instructive in that its residuals show the high energy "curvature" often associated with ULXs (Roberts et al. 2006) and excess emission below $1 \mathrm{keV}$ (Figure 4). Since Galactic black hole binaries do not exhibit such curvature, ULXs are not simply scaled-up versions of ordinary stellar-mass black holes. A broken power law (TBABS*BKNPOWER) with a break energy near $4 \mathrm{keV}$ is an adequate fit (Table 1), but it does not completely remove the excess below $0.4 \mathrm{keV}$ (Figure 4). Likewise, a bremsstrahlung model (TBABS*BREMSS) is a good fit with $k T \approx 3.4 \mathrm{keV}$ but retains this excess. These fits can be improved by adding a cool thermal (APEC) model with fixed $k T=0.1 \mathrm{keV}$; $\delta \chi^{2}=35$ in the broken power-law model for one fewer degree of freedom indicates an improvement at over $95 \%$ significance. In fact, the excess below $0.4 \mathrm{keV}$ is present in all our fits (Figure 4), but the pn and MOS disagree at these energies (see examples in Stuhlinger et al. 2006) so we cannot conclude that the thermal component is physical and do not include it in our reported fits. We also exclude MOS data below $0.4 \mathrm{keV}$. However, including the thermal component does not cause large shifts in model parameters in Table 1.

We attempted some of the disk models recently tried on highquality XMM-Newton spectra in GRD09 and Walton et al. (2011, whose source NGC 4715 ULX1 has a qualitatively similar spectrum). These include a multi-colored disk (MCD) blackbody model (TBABS*DISKPBB), an MCD model with Comptonization (TBABS*(DISKPBB+COMPTT)), a disk irradiated by Comptonized photons (TBABS*DISKIR), and blurred reflection (TBABS*KDBLUR2*REFLIONX). In the Comptonization models, the seed photons are assumed to originate at the inner edge of the disk.

For the pure MCD model we use a " $p$-free" model in which $T(r) \propto r^{-p}$, where $p$ is a free parameter (in the normal MCD model DISKBB, $p$ is frozen at 0.5; Mitsuda et al. 1984). The best fit has an inner disk temperature $T_{\text {in }} \approx 1.6 \mathrm{keV}$ and $p \approx 0.54$, indicating a hot, "slim" disk. The temperature can be lowered to $T_{\text {in }} \sim 1 \mathrm{keV}$ by adding a Comptonization component, but based on simulated spectra the additional component does not significantly improve the fit. Because the spectrum remains disk dominated in either case, we find a degeneracy between a hot, optically thick corona or a cool, optically thin one. GRD09 find that a cool, thick corona is preferred, but we find no global minimum and poor constraints due to the weakness of the component. 


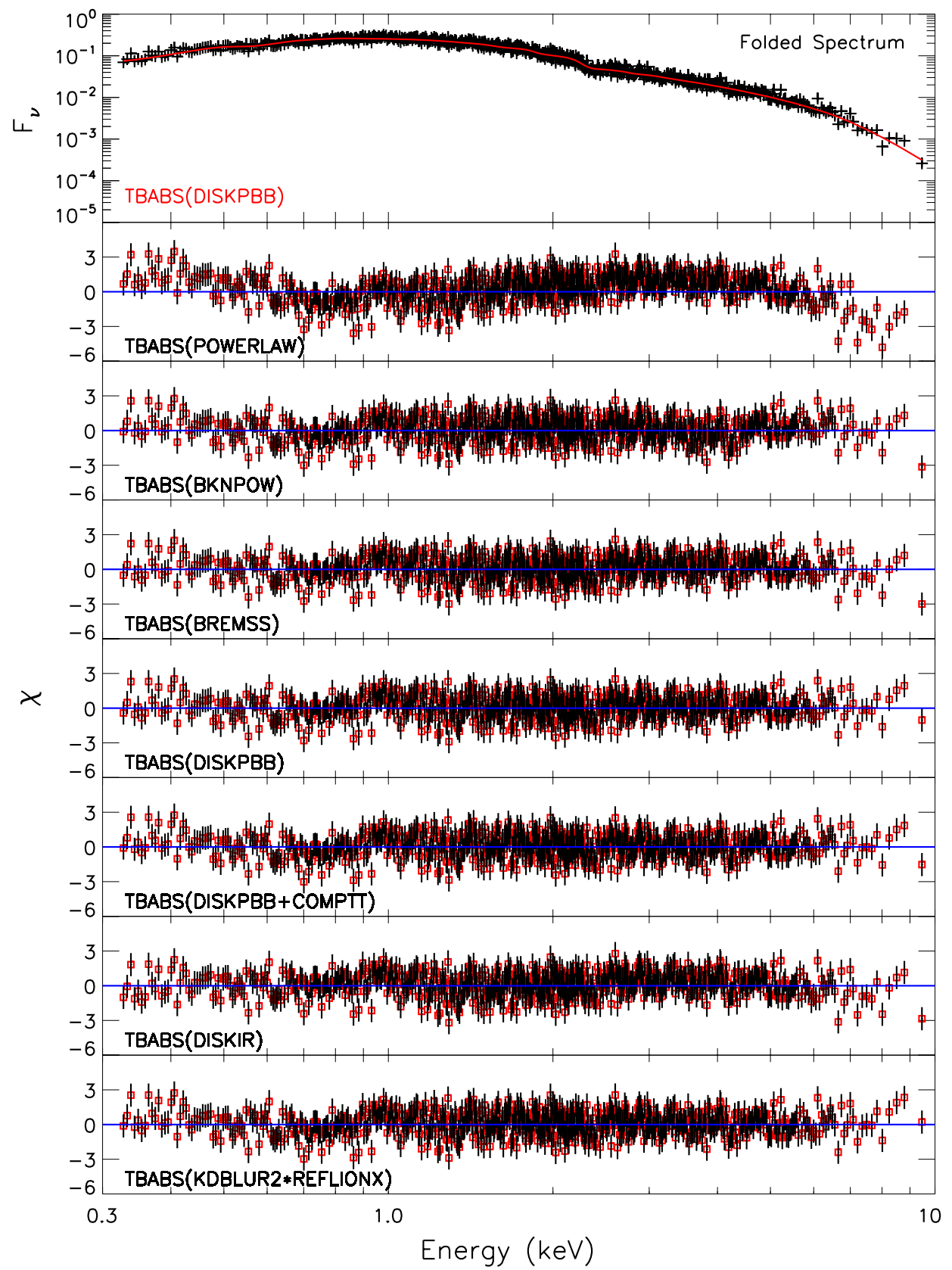

Figure 4. 0.3-10 keV EPIC-pn spectrum (binned to 25 counts) with the best-fit "hot disk" model overplotted. Below are residuals for each model. Note the rightmost residual for the power-law model is at -10 .

(A color version of this figure is available in the online journal.)

We obtain a good fit for a cool disk with a dominant Comptonization component when the disk structure is modified by the absorbing Comptonized photons (DISKIR). In this model, the inner disk is irradiated by Comptonized photons, many of which are "reflected." Some fraction is absorbed and reemitted with a quasi-thermal spectrum, thereby modifying the disk emission (Gierliński et al. 2008). Significant modification only occurs when the Comptonized component is much brighter than the disk emission, naturally forcing fits with cool, relatively dim disks. Thus, this model is expected to be important in the low/hard state-a very different situation from that implied by the MCD model. In order to fit this spectrum,the Compton hump must occur near $2 \mathrm{keV}$, implying a cool corona $\left(k T_{e} \sim 1.4 \mathrm{keV}\right)$ and a cooler disk $\left(T_{\mathrm{in}} \sim 0.4 \mathrm{keV}\right)$. The intrinsic absorption is also substantially lower than in other models.
It is also possible that much of the emission is Comptonized photons "reflected" off the inner edge of the disk with substantial line "blurring" due to relativistic effects near the black hole. Like Walton et al. (2011), we find that a significant amount of blurring is required such that the disk must reach to $1.26 R_{G}$ with an emissivity index $q_{\text {in }}>7$, meaning that the emission is dominated by light from the inner disk.

\section{DISCUSSION}

Despite the high quality of the X-ray spectrum, several very different models produce excellent fits. Thus, additional data at other wavebands or a timing study in the X-rays are required to discriminate between them. We briefly discuss the implications and predictions of each model. 
Table 1

Spectral Models

\begin{tabular}{|c|c|c|c|}
\hline Component & Parameter & Units & Value \\
\hline \multicolumn{4}{|c|}{ TBABS*BREMSS } \\
\hline TBABS & $N_{\mathrm{H}}$ & $10^{21} \mathrm{~cm}^{-2}$ & $1.3 \pm 0.1$ \\
\hline BREMSS & $k T$ & $\mathrm{keV}$ & $3.4 \pm 0.1$ \\
\hline$\chi^{2}(\mathrm{dof})$ & & & $1189.3(1171)$ \\
\hline \multicolumn{4}{|c|}{ TBABS*BKNPOWER } \\
\hline TBABS & $N_{\mathrm{H}}$ & $10^{21} \mathrm{~cm}^{-2}$ & $1.9 \pm 0.1$ \\
\hline BKNPOWER & $\Gamma_{1}$ & & $1.91 \pm 0.04$ \\
\hline & $\Gamma_{2}$ & & $3.2 \pm 0.2$ \\
\hline & BreakE & $\mathrm{keV}$ & $3.7_{-0.3}^{+0.2}$ \\
\hline$\chi^{2}(\mathrm{dof})$ & & & $1217.0(1169)$ \\
\hline \multicolumn{4}{|c|}{ TBABS*DISKPBB (Hot Disk) } \\
\hline TBABS & $N_{\mathrm{H}}$ & $10^{21} \mathrm{~cm}^{-2}$ & $1.5 \pm 0.1$ \\
\hline DISKPBB & $T_{\text {in }}$ & $\mathrm{keV}$ & $1.62_{-0.06}^{+0.05}$ \\
\hline & $p$ & & $0.54 \pm 0.01$ \\
\hline$\chi^{2}$ (dof) & & & $1169.1(1170)$ \\
\hline \multicolumn{4}{|c|}{ TBABS*(DISKPBB+COMPTT) (Hot Disk) } \\
\hline TBABS & $N_{\mathrm{H}}$ & $10^{21} \mathrm{~cm}^{-2}$ & $1.5 \pm 0.1$ \\
\hline DISKPBB & $T_{\text {in }}$ & $\mathrm{keV}$ & $1.1 \pm 0.1$ \\
\hline & $p$ & & $0.54(f)$ \\
\hline СOMPTT & $k T_{e}$ & $\mathrm{keV}$ & $44^{\mathrm{a}}$ \\
\hline & $\tau_{p}$ & & $0.01^{\mathrm{a}, \mathrm{b}}$ \\
\hline or & $k T_{e}$ & $\mathrm{keV}$ & $2.0^{\mathrm{a}, \mathrm{b}}$ \\
\hline & $\tau_{p}$ & & $5.4^{\mathrm{a}}$ \\
\hline$\chi^{2}($ dof $)$ & & & $1166.6(1168)$ \\
\hline \multicolumn{4}{|c|}{ TBABS*DISKIR (Cool Disk) } \\
\hline TBABS & $N_{\mathrm{H}}$ & $10^{21} \mathrm{~cm}^{-2}$ & $0.8_{-0.3}^{+0.5}$ \\
\hline DISKIR & $T_{\mathrm{in}}$ & $\mathrm{keV}$ & $0.38_{-0.03}^{+0.04}$ \\
\hline & $\Gamma$ & & $1.93_{-0.02}^{+0.055}$ \\
\hline & $L_{c} / L_{d}$ & & $7_{-2}^{+4}$ \\
\hline & $k T_{e}$ & $\mathrm{keV}$ & $1.4 \pm 0.3$ \\
\hline & $f_{\text {in }}$ & & 0.1 (f) \\
\hline & $f_{\text {out }}$ & & $0.04^{+0.06 b}$ \\
\hline & $r_{\text {irr }}$ & $r_{\text {in }}$ & $6_{-2}^{+3}$ \\
\hline & $r_{\text {out }}$ & $r_{\text {in }}$ & $10^{5}(\mathrm{f})$ \\
\hline$\chi^{2}($ dof $)$ & & & $1160.8(1166)$ \\
\hline \multicolumn{4}{|c|}{ TBABS*KDBLUR2*REFLIONX (Blurred Reflection) } \\
\hline TBABS & $N_{\mathrm{H}}$ & $10^{21} \mathrm{~cm}^{-2}$ & $2.1 \pm 0.2$ \\
\hline \multirow[t]{6}{*}{ KDBLUR2 } & $R_{\text {in }}$ & $R_{G}$ & $1.26^{+0.08 b}$ \\
\hline & $R_{\text {out }}$ & $R_{G}$ & 400 (f) \\
\hline & $i$ & deg & $30 \pm 11$ \\
\hline & $q_{\text {in }}$ & & $8_{-1}^{+2}$ \\
\hline & $q_{\text {out }}$ & & 3.0 (f) \\
\hline & $R_{\text {break }}$ & $R_{G}$ & 20.0 (f) \\
\hline \multirow[t]{3}{*}{ REFLIONX } & $\Gamma$ & & $1.73 \pm 0.06$ \\
\hline & $\xi$ & $\operatorname{erg~} \mathrm{cm} \mathrm{s}^{-1}$ & $>6000^{\mathrm{b}}$ \\
\hline & $A_{\mathrm{Fe}}$ & & $8 \pm 3$ \\
\hline$\chi^{2}(\mathrm{dof})$ & & & $1202.34(1167)$ \\
\hline
\end{tabular}

Notes. All fits incorporate a TBABS component frozen at the Galactic $N_{\mathrm{H}}=$ $6.5 \times 10^{20} \mathrm{~cm}^{-2}$. Additional absorption is listed here in units of $10^{21} \mathrm{~cm}^{-2}$. Errors are quoted at the $90 \%$ confidence interval based on the XSPEC task steppar.

a These parameters are not well constrained and we do not quote errors. See the text.

b These parameters are near the boundary of parameter space.

A hot disk. The hot disk model implies a black hole mass of less than $40 M_{\odot}$ (e.g., Soria \& Kuncic 2008), hence suggesting super-Eddington accretion. Similar hot-disk ULXs are explained as stellar-mass black holes by Winter et al. (2006), and the recent appearance of the source makes sense if, as expected, super-Eddington accretion is a transient phenomenon. The Chandra non-detection places a prior limit of $L_{X}<$ $10^{37} \mathrm{erg} \mathrm{s}^{-1}$, which is consistent with a low/hard state in a stellar-mass black hole but quiescence in an IMBH. The excellent fit with a small number of parameters is also a point in favor of the pure MCD model, and a disk-dominated spectrum is consistent with an extreme version of the high/soft state seen in Galactic black holes (McClintock \& Remillard 2006, GRD09).

The unabsorbed model luminosity is $L_{X}=(2.1 \pm 0.3) \times$ $10^{40} \mathrm{erg} \mathrm{s}^{-1}$, significantly higher than other disk-dominated ULXs (Swartz et al. 2004, 2003; Roberts et al. 2002) and modestly higher than the brightest hot-disk sources in Winter et al. (2006). This luminosity exceeds the Eddington limit by 2.5 times for a $100 M_{\odot}$ black hole. Hence, it does not easily fit into the "sequence" proposed by GRD09, in which disk-dominated sources are sub-Eddington accretors on the extreme tail of the stellar-mass black hole mass distribution. As the source maintains the same hardness ratio seen in the XMM-Newton spectra during Swift monitoring, there may be substantial scatter in $L_{X}-T_{\text {in }}$ plots (cf. Miller et al. 2004). If the source is thermally dominated (the simplest model with an excellent fit), it would join only a few other ULXs seen in this state (Feng \& Kaaret 2010; Jin et al. 2010; Servillat et al. 2011).

In the case of super-Eddington accretion we expect to see powerful outflows. Since the outburst began at most five years ago, these outflows would be within $\sim 1$ pc of the source. We might therefore expect to see absorption signatures in a high quality RGS spectrum, although the source geometry is unknown. Detection of such an outflow would place a strong constraint on the mechanical luminosity, an important parameter in models of super-Eddington accretion (e.g., Begelman 2002; Dotan \& Shaviv 2011). If super-Eddington accretion in this system is episodic, broad or multiple absorption features would be expected.

A cool disk. If the hot disk model is similar to an extreme version of the high state seen in Galactic black holes, a cool disk may correspond roughly to the low/hard state (Gierliński et al. 2008). In the irradiated disk model, the spectrum is dominated by a cool, Comptonized component with the subjacent disk component dominant at low energies. This model presumes strong reflection, but the absence of a strong $\mathrm{Fe} \mathrm{K} \alpha$ line is consistent with the low corona temperature $\left(k T_{e} \approx 1.4 \mathrm{keV}\right)$. The emission comes almost exclusively from the very inner region of the disk, which itself is cool $\left(T_{\text {in }} \lesssim 0.4 \mathrm{keV}\right)$. If this disk extends to the innermost stable circular orbit, its temperature would indicate an IMBH (and we would expect the luminosity to vary as $L_{X} \propto T_{\text {in }}$ in future observations). However, the disk may be truncated at large radii outside of a large corona or outflow (further discussed in Feng \& Soria 2011), in which case the disk temperature cannot be used to infer a mass.

There are a few objections to this model. First, a cool disk component is only a good fit when irradiated, otherwise a hot disk is required (even the "cooler" hot disk has $T_{\text {in }} \sim 1 \mathrm{keV}$ ). If the disk were intrinsically cool, we would expect a nonirradiated cool disk to at least be competitive with the hot disk. Second, if the black hole is in the low/hard state, the prior X-ray non-detections suggest that it was previously quiescent; even the naked disk in this model should have been detected.

Fortunately, this model can be falsified in a few ways. The intrinsic absorption in this fit is small compared to the others, so if an optical counterpart could be established and 
its intrinsic color determined, a column could be measured. We would also expect to see variability like that in Galactic low/hard states (and potentially quasi-periodic oscillations as in Strohmayer \& Mushotzky 2009), so long-term X-ray monitoring can potentially discriminate between a high and a low state.

Blurred reflection. In contrast to the other models, the blurred reflection model fits the spectrum assuming it is dominated by reflection of Comptonized photons near the inner edge of the disk, with relativistic effects smearing out the strong emission lines. This model predicts a Compton hump in the 10-30 keV range, so observations above $10 \mathrm{keV}$ could easily distinguish between this model and the others. Whereas many ULXs are in galaxies with nuclear sources of comparable or greater brightness, NGC 891 ULX1 is presently the brightest $\mathrm{X}$-ray source in the galaxy. As in the irradiated disk model, blurred reflection suggests the low/hard state. The caveats of the physical interpretation here are discussed in detail in Walton et al. (2011), but we note here that this model requires suppression of the disk emission.

Like other ULX spectra, the mostly featureless continuum of NGC 891 ULX1 admits several different scenarios, suggesting observations in other wavebands or in the time domain are necessary to untangle the origin of the X-rays. Given that the outburst is recent and preceded by at least a 20 year lull, a search for an ionized nebula like those seen around other nearby, bright ULXs (Pakull \& Mirioni 2002) is of interest. Such a nebula would point to recurrent ultraluminous activity, and its size and power may allow measurement of the duty cycle.

\section{SUMMARY}

A recent $X M M-N e w t o n$ observation revealed a bright new source toward NGC 891. At this point, the most natural explanation is that it is a source within the galaxy, in which case its flux and the absence of detections in other wavelengths make a ULX the best explanation. The XMM-Newton spectrum is morphologically similar to disk-dominated sources in the GRD09 "sequence" of ULXs and is indeed fit well by a hot MCD disk. However, several very different physical scenarios produce good fits to the spectrum, including an irradiated cool disk with strong but cool Comptonization and a blurred reflection model. In the hot-disk model, NGC 891 ULX1 has a mass less than $40 M_{\odot}$ and therefore accretion rates over five times Eddington. If so, the recent ignition suggests that a search for outflows would be a worthwhile test of super-Eddington models.

The authors thank the referee for catching errors and making comments that significantly improved the paper, as well as
R. Mushotzky for helpful comments and A. Filippenko for information regarding SN monitoring. E.H.K. gratefully acknowledges support from NASA ADAP grant 061951.

Facilities: XMM, EVLA, Swift (XRT), CXO (ACIS), Mayall, HST (ACS)

\section{REFERENCES}

Arnaud, K. A. 1996, in ASP Conf. Ser. 101, Astronomical Data Analysis Software and Systems V, ed. G. H. Jacoby \& J. Barnes (San Francisco, CA: ASP), 17

Begelman, M. C. 2002, ApJ, 568, L97

Colbert, E. J. M., \& Mushotzky, R. F. 1999, ApJ, 519, 89

de Vaucouleurs, G., de Vaucouleurs, A., Corwin, H. G., Jr., et al. 1991, Third Reference Catalogue of Bright Galaxies, ed. N. G. Roman et al. (New York: Springer)

Dotan, C., \& Shaviv, N. J. 2011, MNRAS, 413, 1623

Fabbiano, G. 1989, ARA\&A, 27, 87

Feng, H., \& Kaaret, P. 2010, ApJ, 712, L169

Feng, H., \& Soria, R. 2011, New Astron. Rev., 55, 166

Garcia-Burillo, S., \& Guelin, M. 1995, A\&A, 299, 657

Gierliński, M., Done, C., \& Page, K. 2008, MNRAS, 388, 753

Gladstone, J. C., Roberts, T. P., \& Done, C. 2009, MNRAS, 397, 1836

Jin, J., Feng, H., \& Kaaret, P. 2010, ApJ, 716, 181

Kalberla, P. M. W., Burton, W. B., Hartmann, D., et al. 2005, A\&A, 440, 775

King, A. R., Davies, M. B., Ward, M. J., Fabbiano, G., \& Elvis, M. 2001, ApJ, 552, L109

Li, J., Kastner, J. H., Prigozhin, G. Y., et al. 2004, ApJ, 610, 1204

McClintock, J. E., \& Remillard, R. A. 2006, in Black Hole Binaries, ed. W. H. G. Lewin \& M. van der Klis (Cambridge: Cambridge Univ. Press), 157

Merloni, A., Heinz, S., \& di Matteo, T. 2003, MNRAS, 345, 1057

Miller, J. M., Fabian, A. C., \& Miller, M. C. 2004, ApJ, 614, L117

Mitsuda, K., Inoue, H., Koyama, K., et al. 1984, PASJ, 36, 741

Pakull, M. W., \& Mirioni, L. 2002, arXiv:astro-ph/0202488

Protassov, R., van Dyk, D. A., Connors, A., Kashyap, V. L., \& Siemiginowska, A. 2002, ApJ, 571, 545

Roberts, T. P., Stobbart, A.-M., Goad, M. R., et al. 2006, in Proc. The X-ray Universe 2005, ed. A. Wilson (ESA SP-604; Paris: ESA), 427

Roberts, T. P., Warwick, R. S., Ward, M. J., \& Murray, S. S. 2002, MNRAS, 337,677

Servillat, M., Farrell, S. A., Lin, D., et al. 2011, ApJ, 743, 6

Shang, Z., Brotherton, M. S., Wills, B. J., et al. 2011, ApJS, 196, 2

Soria, R., \& Kuncic, Z. 2008, Adv. Space Res., 42, 517

Spergel, D. N., Bean, R., Doré, O., et al. 2007, ApJS, 170, 377

Strohmayer, T. E., \& Mushotzky, R. F. 2009, ApJ, 703, 1386

Stuhlinger, M., Altieri, B., Esquej, M. P., et al. 2006, in The X-ray Universe 2005, ed. A. Wilson (ESA SP-604; Paris: ESA), 937

Swartz, D. A., Ghosh, K. K., McCollough, M. L., et al. 2003, ApJS, 144, 213 Swartz, D. A., Ghosh, K. K., Tennant, A. F., \& Wu, K. 2004, ApJS, 154, 519

Temple, R. F., Raychaudhury, S., \& Stevens, I. R. 2005, MNRAS, 362, 581

Tully, R. B., Rizzi, L., Shaya, E. J., et al. 2009, AJ, 138, 323

van der Kruit, P. C., \& Searle, L. 1981, A\&A, 95, 116

Walton, D. J., Gladstone, J. C., Roberts, T. P., et al. 2011, MNRAS, 414, 1011

Wilms, J., Allen, A., \& McCray, R. 2000, ApJ, 542, 914

Winter, L. M., Mushotzky, R. F., \& Reynolds, C. S. 2006, ApJ, 649, 730

Zezas, A., \& Fabbiano, G. 2002, ApJ, 577, 726 